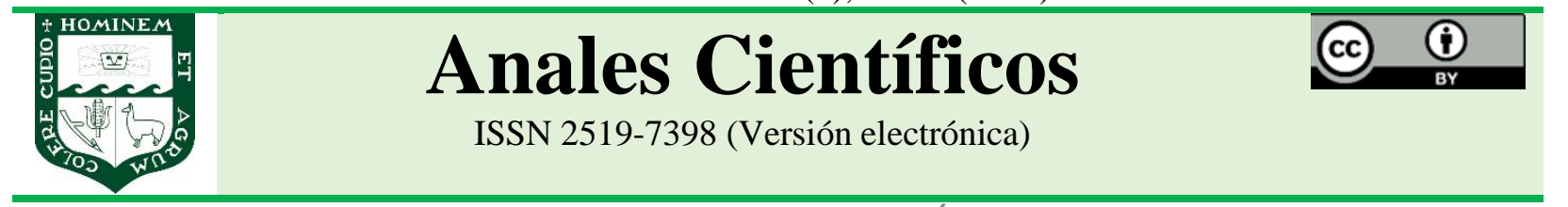

ARTÍCULO ORIGINAL - RESEARCH ARTICLE

http://dx.doi.org/10.21704/ac.v82i1.1739

\title{
COSTO DE OPORTUNIDAD DEL SECTOR AGRÍCOLA COMERCIAL EXPORTADOR EN EL PERÚ, 1998-2017
}

\section{Opportunity cost of the exporting commercial agricultural sector in Peru, 1998-2017}

\author{
Juan F. Magallanes Díaz ${ }^{1 *(1)}$; Carlos F. Camasi Montes ${ }^{1}(\mathbb{0}$ \\ ${ }^{1}$ Facultad de Economía y Planificación, Universidad Nacional Agraria La Molina, 15024, Lima, Perú. \\ * E-mail: jmagallanes@ @lamolina.edu.pe
}

Recibido: 18/12/2019; Aceptado: 11/05/2021; Publicado: 31/08/2021

\begin{abstract}
The objective of the investigation was to determine the opportunity cost in the commercial export agricultural sector, based on in the economic foundations for Peru, period 1998-2017. The methodology of the research was explanatory and was carried out under a longitudinal non-experimental design, with the application of the capital asset price model (CAPM) for determining the opportunity cost of the exporting commercial agricultural sector of Peru, in the period 1998-2017. Data were processed from the share price indices of 19 agricultural companies listed on the Lima Stock Exchange. The results showed the relationship of market performance variables and systematic risk (Beta) in the determination of the opportunity cost of the commercial agricultural exporting sector, obtaining a value greater than $29 \%$. In conclusion, there is viability of agricultural projects and businesses that previously were not because much higher rates were applied, managed by the banking system in Peru.
\end{abstract}

Keywords: opportunity cost; market yields; systematic risk

\section{RESUMEN}

El objetivo de la investigación fue determinar el costo de oportunidad en el sector agrícola comercial exportador, argumentado en los fundamentos económicos para el Perú, periodo 1998-2017. La metodología de la investigación ha sido de carácter explicativa y se realizó bajo un diseño no experimental longitudinal, con la aplicación del modelo de precio del activo de capital (CAPM, siglas en inglés) en la determinación del costo de oportunidad del sector agrícola comercial exportador del Perú, en el periodo 1998-2017. Se procesaron datos de los índices de precios de las acciones de 19 empresas agrícolas que cotizan en la Bolsa de Valores de Lima. Los resultados muestran la relación de las variables rendimiento del mercado y riesgo sistemático (Beta) en la determinación del costo de oportunidad del sector agrícola comercial exportador, obteniendo un valor superior al 29\%. En conclusión, existe viabilidad de proyectos y negocios agrícolas que antes no lo eran porque se aplicaban tasas mucho más elevadas, manejadas por el sistema bancario en el Perú.

Palabras clave: costo de oportunidad; rendimiento de mercado; riesgo sistemático

Forma de citar el artículo (Formato APA):

Magallanes, F., \& Camasi, C. (2021). Costo de oportunidad del sector agrícola comercial exportador en el Perú, 1998-2017.

Anales Científicos. 82(1), 33-41. http://dx.doi.org/10.21704/ac.v82i1.1739 
Autor de correspondencia (*): Juan Magallanes Díaz. Email: jmagallanes@lamolina.edu.pe

(c) Los autores. Publicado por la Universidad Nacional Agraria La Molina.

This is an open access article under the CC BY

\section{INTRODUCCIÓN}

El sector agrícola peruano, con los proyectos promovidos por el Ministerio de Agricultura y Riego (MINAGRI) actualmente MIDAGRI, vienen logrando un impacto positivo sobre la productividad y la liquidez financiera en el sector, incentivando la entrada en escena de nuevas inversiones agropecuarias. En efecto, en el 2015 se exportaron productos agrícolas por el valor de US\$5 500 millones, en el 2016 por un valor de US\$6 000 millones, en el 2017 por un valor de US\$6 500 millones y se estima que estas superen los US\$8 000 millones en los próximos cinco años (MINAGRI, 2017).

Debido al riesgo de inversión en este sector se requiere una adecuada evaluación económica y financiera. En este aspecto, una de las preocupaciones constantes es determinar el costo de oportunidad que debería utilizarse para descontar cualquier flujo futuro de rentas netas que genere cualquier inversión en la agricultura y comprobar si el proyecto agroexportador que se va a realizar es bueno o no en términos monetarios (Sarmiento, 2008).

El costo de oportunidad del capital se refiere a la tasa de retorno exigida a una inversión realizada, sea en un proyecto o en una empresa, para compensar la variabilidad del riesgo y el costo financiero de los recursos por préstamos o créditos. Esto indica que se deben tener en cuenta variables complejas tales como riesgo, tasas de interés, políticas fiscales, entre otras. En el ámbito académico, se acostumbra a asemejar el costo de oportunidad al costo de capital asumiendo que existen condiciones de equilibrio perfecto en la economía, aunque la realidad de las empresas y proyectos aún dista del equilibrio perfecto teórico (Vélez-Pareja, 2011).

El modelo CAPM, costo de oportunidad, por su nombre en inglés Capital Asset Pricing Model y el cálculo de los coeficientes Beta (riesgo sistemático que no puede eliminarse de los portafolios de inversión) o modelo de valoración de activos de capital, permite asignar, calcular, valorar el precio y comportamiento de activos financieros variables (Markowitz, 1952). CAPM (costo de oportunidad) es la metodología tradicional para estimar el costo de oportunidad de capital. Se basa en premisas de mercado de capitales perfectamente eficientes (información perfecta y diversificación racional de portafolio); en términos generales, parte de la base de que el costo de capital tiene dos componentes: un rendimiento mínimo que se debe compensar al inversionista por el valor del dinero en el tiempo (tasa libre de riesgo) y una prima por el riesgo no diversificable del instrumento Beta (Fernández, 2008).

Lo precedente, lleva al problema de esclarecer los fundamentos en la determinación del costo de oportunidad del sector agrícola comercial transable, pues de no contar con una tasa de costo de oportunidad fundamentada, no se obtendría una adecuada valoración de cualquier flujo de rentas futuras generadas en el sector.

El objetivo principal del estudio fue determinar el costo de oportunidad para el sector agrícola comercial exportador del Perú, argumentado en fundamentos económicos aplicados al sector y para el periodo 1998-2017. Para lograr el objetivo planteado, se debe buscar (i) determinar el grado de incidencia del rendimiento de mercado en el riesgo sistemático (Beta) del sector agrícola comercial exportador para el Perú, periodo 1998-2017; (ii) determinar el grado e incidencia del riesgo sistemático (Beta) en el costo de oportunidad del sector agrícola comercial exportador para el Perú, periodo 1998- 2017.

\section{MATERIALES Y MÉTODOS}

La investigación se realizó en el ámbito geográfico de Lima, Perú, periodo 1998-2017. La metodología utilizada fue de carácter cuantitativa, explicativa, no experimental, y de corte longitudinal. 
Para la obtención de la tasa de costo de oportunidad se utilizó el modelo Capital Asset Pricing Model $(\mathbf{C A M P}=\mathbf{K s}=\mathbf{C O K})$, modelo desarrollado por Sharpe, Lintner y Mossin (Kristjanpoller et al. 2010), es necesario los valores de una tasa libre de riesgo (Rf), de la tasa de rendimiento de mercado (Rm) de los activos financieros del sector agrario, el coeficiente $\beta$ como indicador de riesgo sistemático, y el coeficiente $\Theta$ como indicador del riesgo país.

$\mathrm{Ks}=\mathrm{Rf}+\beta(\mathrm{Rm}-\mathrm{Rf})+\Theta$

Se trabajó con datos obtenidos en un periodo de 20 años (1998-2017), información extraída del Índice General de la Bolsa de Valores de Lima (IGBVL), y de un Índice Agrario del Perú (INAG) periodo 19982017, este último integra el índice de precios de las acciones de un total de 19 empresas agrícolas y agroindustriales que cotizan en bolsa, dedicados principalmente a bienes agrícolas transables con el sector externo, datos que se encuentran registrados en forma diaria y mensual, siendo considerados el total de estas 19 empresas el universo y muestra del estudio, con 6900 registros de datos diarios los cuales se integraron y ordenaron en datos mensuales, distribuyéndose de la siguiente manera: (i) Muestra de entrenamiento: se utilizó las 6900 observaciones diarias registradas, (ii) Muestra de validación: se utilizó las 330 observaciones mensuales.

Con los análisis de datos y desarrollo del IGBVL y del índice del sector Agrario (INAG), se homogenizaron indicadores estadísticos de diferentes años base, con los empalmes estadísticos necesarios. Se determinaron las variables para el riesgo sistemático y determinación de la beta sectorial. Se aplicó la metodología para determinar el costo de oportunidad del sector agrícola transable. A continuación, se comparó el resultado del costo de oportunidad agrícola comercial exportador por el modelo CAPM, con el costo de oportunidad que actualmente viene aplicándose en forma empírica al sector agrícola comercial exportador en el Perú.

\section{RESULTADOS Y DISCUSIÓN}

Considerando el modelo planteado y las variables determinantes, se procedió a la determinación de los valores de estas últimas, y llegar finalmente a la determinación del COK en el sector agrícola, cuyos resultados siguen a continuación.

\section{Determinación de la tasa libre de riesgo $\mathbf{R f}$}

como se muestra en la Tabla 1, para asignar el valor de la tasa libre de riesgo, se tomó el promedio del rendimiento de los Bonos del Tesoro Perú MEF, o conocidos como Bonos Soberanos de los últimos 20 años (1998-2017), considerado teóricamente como la tasa de rendimiento de riesgo cero; en este caso el valor promedio obtenido es de $6,89 \%$ para ese período.

Tabla 1. Tasa Libre Riesgo del Perú de los últimos veinte años (Banco Central de Reserva del Perú, 2017).

\begin{tabular}{cccc}
\hline Año & Rf & Año & Rf \\
\hline 1998 & $11,2 \%$ & 2008 & $6,4 \%$ \\
1999 & $11,7 \%$ & 2009 & $6,2 \%$ \\
2000 & $11,7 \%$ & 2010 & $4,9 \%$ \\
2001 & $11,5 \%$ & 2011 & $4,7 \%$ \\
2002 & $10,7 \%$ & 2012 & $3,4 \%$ \\
2003 & $8,3 \%$ & 2013 & $3,9 \%$ \\
2004 & $7,9 \%$ & 2014 & $4,2 \%$ \\
2005 & $6,7 \%$ & 2015 & $4,1 \%$ \\
2006 & $6,6 \%$ & 2016 & $3,8 \%$ \\
2007 & $6,0 \%$ & 2017 & $3,8 \%$ \\
\hline
\end{tabular}

\section{Rm = Rendimiento o Retornos de mercado (Rend. IGBVL)}

El mercado hace referencia al promedio del valor de las acciones del mercado bursátil de Lima, o más conocido como el Índice General de la Bolsa de Valores de Lima (IGBVL) en forma anual, y los registrados al final de cada uno de los 20 años de análisis. El rendimiento o retornos del IGBVL es la variación porcentual anual, cuyo valor promedio Rm obtenido es de $22,58 \%$ para este período (Tabla 2). También, se presentan los índices y rendimientos de los valores vinculados al sector agrario. 
Tabla 2. Rendimiento anual Agrario y de la Bolsa de Valores de Lima (BVL, 2017).

\begin{tabular}{|c|c|c|c|c|}
\hline & \multicolumn{2}{|c|}{ Índices } & \multicolumn{2}{|c|}{ Retornos } \\
\hline & Agrario & IGBVL & Agrario & IGBVL \\
\hline Dic-98 & 0,9894 & 0,7278 & & \\
\hline Dic-99 & 10,000 & 10,000 & 0,010720 & 0,374053 \\
\hline Dic-00 & 0,5138 & 0,6583 & $-0,486163$ & $-0,341670$ \\
\hline Dic-01 & 0,5439 & 0,6409 & 0,058548 & $-0,026448$ \\
\hline Dic-02 & 0,5705 & 0,7583 & 0,048927 & 0,183195 \\
\hline Dic-03 & 0,5542 & 13,266 & $-0,028562$ & 0,749348 \\
\hline Dic-04 & 0,8661 & 20,214 & 0,562630 & 0,523749 \\
\hline Dic-05 & 12,274 & 26,162 & 0,417168 & 0,294271 \\
\hline Dic-06 & 41,889 & 70,192 & $2,412,915$ & $1,682,951$ \\
\hline Dic-07 & 78,709 & 95,473 & 0,878991 & 0,360177 \\
\hline Dic-08 & 27,448 & 38,400 & $-0,651276$ & $-0,597789$ \\
\hline Dic-09 & 57,816 & 77,181 & $1,106,408$ & $1,009,911$ \\
\hline Dic-10 & 103,274 & 127,342 & 0,786244 & 0,649908 \\
\hline Dic-11 & 89,151 & 106,089 & $-0,136754$ & $-0,166902$ \\
\hline Dic-12 & 87,901 & 112,387 & $-0,014017$ & 0,059365 \\
\hline Dic-13 & 49,125 & 85,824 & $-0,441127$ & $-0,236348$ \\
\hline Dic-14 & 45,126 & 80,598 & $-0,081416$ & $-0,060896$ \\
\hline dic-15 & 21,059 & 53,654 & $-0,533333$ & $-0,334299$ \\
\hline Dic-16 & 45,126 & 80,598 & $1,142,857$ & 0,502176 \\
\hline Dic-17 & 21,059 & 53,654 & $-0,533333$ & $-0,334299$ \\
\hline
\end{tabular}

\section{Rm-Rf = Prima de riesgo del mercado}

La prima de riesgo del mercado es el spread o diferencia entre el promedio del rendimiento de las acciones totales negociadas registradas en el Índice General de la Bolsa de Valores de Lima IGBVL (Rm), menos el rendimiento promedio de los bonos del tesoro público o bonos soberanos (Rf), ambos para el mismo período de 20 años, cuyo resultado obtenido fue de $22,58-6,89=15,70 \%$.

\section{$\beta=$ Coeficiente beta o riesgo sistemático}

Coeficiente numérico que relaciona la proporcionalidad de cambio o sensibilidad del rendimiento de los valores agrícolas (índice agrario), ante variaciones en el rendimiento del mercado o del índice general de la BVL, esto significa en términos estadísticos obtener un coeficiente de cambio mediante una regresión lineal simple, o también de obtener la relación entre la covarianza de ambas variables entre la varianza del rendimiento del mercado Rm, de acuerdo a la fórmula estadística que sigue a continuación.

$(\beta)=$ Covarianza $\quad($ RINAG, $\quad$ RIGBVL)/Varianza(RIGBVL)

Para determinar el Beta del sector agrícola comercial exportador, se generaron los retornos del Índice General de la Bolsa de Valores de Lima y el retorno del Índice Agrario, periodo 1998-2017.

\section{RIGBVL= (IGBVLt / IGBVLt-1)-1}

RIGBVL: Retornos del Índice General de la Bolsa de Valores de Lima.

IGBVLt: Índice General de la Bolsa de Valores de Lima en la fecha $t$.

IGBVLt-1: Índice General de la Bolsa de Valores de Lima en la fecha $t-1$.

RINAG $=($ INAGt $/$ INAGt- 1$)-1$

RINAG: Retornos del Índice Agrario.

INAGt: Índice Agrario en la fecha t.

INAGt-1: Índice Agrario en la fecha t-1.

Luego se procedió a determinar la covarianza de los retornos RINAG, RIGBVL, y también a determinar la varianza del RIGBVL, del periodo 1998-2017, se relacionaron ambos valores $y$ se obtuvo el coeficiente beta (Tabla 3).

Tabla 3. Cálculo de Beta.

\begin{tabular}{lr}
\hline Covarianza (Agrario, IGBVL) & $\mathbf{0 , 0 0 9 9 4 5}$ \\
Varianza (IGBVL) & $\mathbf{0 , 0 0 7 9 5 6}$ \\
\hline Beta & 1,25 \\
\hline
\end{tabular}

El cálculo del coeficiente Beta $(\beta)$ para el sector agrícola comercial por el método del ajuste de regresión lineal (pendiente de la recta ajustada), ofrece el mismo resultado de 1,25 (Figura 1).

Luego, se procedió a realizar el análisis de consistencia en la determinación del ( $\beta$ ) Beta del sector agrícola comercial periodo 1998-2017, para esto se tomaron los mismos datos de la Tabla 2. 


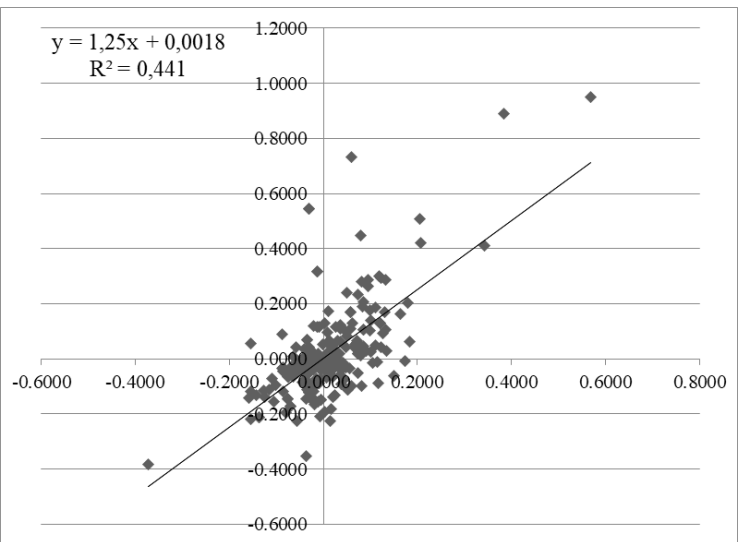

Figura 1. ( $\beta$ ) Beta del sector agrícola comercial.

El tipo de data utilizada es de serie de tiempo porque es una secuencia de datos medidas en determinados momentos del tiempo, para este caso, de 1998 hasta el año 2017.

Las variables utilizadas para el análisis de consistencia en la determinación de la beta del sector agrícola han sido los siguientes:

RINAG: Agrario (Retorno Agrarios Anual): variable dependiente.

RIGBVL: (Retorno del Índice General Bolsa Valores de Lima): variable independiente.

Función múltiple:

$$
R_{N A G:}=\beta_{1}+\beta_{2} R_{G B V L}+u_{i}
$$

B 1, $\beta 2=$ coeficientes desconocidos de regresión $\mu \mathrm{i}=$ término de error aleatorio de la regresión

Con lo anterior estamos atendiendo el primer objetivo de determinar el grado de incidencia del rendimiento de mercado en el riesgo sistemático (Beta) del sector agrícola comercial exportador, en cuanto a la consistencia de este último parámetro se refiere.

En este caso, lo que opera como prueba de hipótesis alterna, la variable regresora RIGBVL es consistente y explicó el comportamiento de la variable regresada, por las bondades y significancia estadística obtenida. Se encontró que la variable RIGBVL ejerce influencia en la variable RINAG, tal como se muestra en la Tabla 4.

\section{$H 1: \beta \_j \neq 0 \quad ; j=2$,}

Tabla 4. Regresión lineal de la variable RINAG (BVL, 2017).

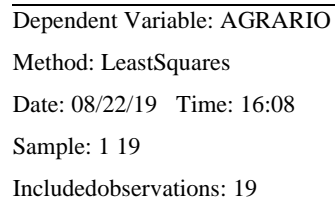

\begin{tabular}{lrlrr}
\hline \hline Variable & Coefficient & Std. Error & t-Statistic & Prob. \\
& & & & \\
\hline \hline C & $-0,037214$ & 0,079405 & $-0,468$ & 0,6453 \\
IGBVL & 1,239599 & 0,135326 & 9,160 & 0,0000 \\
\hline \hline R-squared & 0,831529 & Mean dependentvar & 0,242704 \\
Adjusted R-squared & 0,821619 & S.D. dependentvar & 0,756388 \\
S.E. ofregression & 0,319462 & Akaike infocriterion & 0,654943 \\
Sum squaredresid & 1,734950 & Schwarz criterion & 0,754358 \\
Log likelihood & $-4,221957$ & Hannan-Quinn criter. & 0,671768 \\
F-statistic & 83,90768 & Durbin-Watson stat & 1,438942 \\
Prob(F-statistic) & 0,000000 & & \\
\hline
\end{tabular}

Con un nivel de probabilidad menor al 5\%, se indicó la evidencia estadística para rechazar la hipótesis nula, por lo tanto, se pudo afirmar que la variable RIGBVL influye en cambios en la variable RINAG.

\section{Significancia Conjunta de la variable RINAG}

$\mathrm{R} 2=0,831529$, es muy cercano a la unidad, lo que significó que la variable regresora RIGBVL explicó en un $83,15 \%$ a la variable dependiente RINAG.

$\mathrm{S}=0,319462$, como el error estándar de la regresión fue menor al $15 \%$ del promedio de la variable independiente (AGRARIO=0,242704), se encontró que la regresión tiene un buen ajuste, las variables independientes interpretaron bien a la variable regresada.

\section{$\boldsymbol{\theta}=$ Riesgo país}

El riesgo país debe reflejar el grado de riesgo probable de incumplimiento que un país determinado pueda honrar su deuda internacional, existen diversas formas de medirla, convencionalmente es por la diferencia que hay entre la tasa de rendimiento de los Bonos del Tesoro del Perú menos la tasa de Rendimiento de los Bonos 
del Tesoro de los Estados Unidos, que pueden ser medidos en porcentaje o en puntos.

En la Tabla 5 se presenta el diferencial en porcentaje, para el período 1998 - 2017, cuanto mayor sea ese valor, mayor es el spread, mayor es el riesgo país.

Tabla 5. Valores del indicador Riesgo País para el Perú, 1998-2017 (Banco Central de Reserva del Perú, 2017).

\begin{tabular}{cccc}
\hline Año & $\begin{array}{c}\text { Riesgo País } \\
\text { Perú }\end{array}$ & Año & $\begin{array}{c}\text { Riesgo País } \\
\text { Perú }\end{array}$ \\
\hline 1998 & $6,55 \%$ & 2008 & $2,73 \%$ \\
1999 & $6,04 \%$ & 2009 & $2,92 \%$ \\
2000 & $5,68 \%$ & 2010 & $1,72 \%$ \\
2001 & $6,51 \%$ & 2011 & $1,91 \%$ \\
2002 & $6,11 \%$ & 2012 & $1,57 \%$ \\
2003 & $4,29 \%$ & 2013 & $1,59 \%$ \\
2004 & $3,64 \%$ & 2014 & $1,62 \%$ \\
2005 & $2,39 \%$ & 2015 & $2,01 \%$ \\
2006 & $1,86 \%$ & 2016 & $2,00 \%$ \\
2007 & $1,38 \%$ & 2017 & $1,45 \%$ \\
\hline
\end{tabular}

Tomando los datos observados, el valor promedio de riesgo país para este período arrojó un valor de $3,20 \%$. Habiendo obtenido los valores de las variables determinantes del modelo CAPM, se procedió a la determinación del $\mathrm{COK}$ en el sector agrícola.

\section{Ks = Costo de Oportunidad del Sector Agrícola, COAgricola}

En esta oportunidad, sigue la integración de los resultados parciales obtenido anteriormente para cada variable, en el modelo CAPM antes presentado, y obtener con ello el costo de oportunidad para el sector agrícola comercial, siendo el resultado final de $29,7 \%$ de $\mathrm{CO}_{\text {Agricola. }}$

El cálculo final y complementario se refiere al análisis de consistencia en la determinación del Costo de Oportunidad del Agrícola por sus variables determinantes, y para los 20 años de observación, tal como se presentan los datos en la Tabla 7.

Tabla 6. Datos componentes del Costo de Oportunidad del Sector Agrícola Comercial.

\begin{tabular}{|c|c|c|c|c|c|}
\hline \multirow{2}{*}{\multicolumn{2}{|c|}{$\begin{array}{l}\text { Rendimiento Libre de Riesgo } \\
\text { Rendimiento de Mercado }\end{array}$}} & $\mathrm{Rf}$ & $6,89 \%$ & Promedio Rend.Bonos MEF a 20 años & www.mef.gob.pe \\
\hline & & $\mathrm{Rm}$ & $22,58 \%$ & Promedio BVL últimos 20 años & $\underline{\text { www.bvl.com }}$ \\
\hline \multicolumn{2}{|l|}{ Beta } & $\beta$ & 1,25 & Sobre retornos diarios últimos 20 años & www.bvl.com \\
\hline \multicolumn{2}{|l|}{ Riesgo País } & $\mathrm{RP}$ & $3,20 \%$ & Promedio quincenal último 20 años & www.bcrp.gob.pe \\
\hline $\mathrm{CO}_{\text {Agrícola }}$ & $29,70 \%$ & & & & \\
\hline
\end{tabular}

El tipo de data utilizada es de serie de tiempo porque es una secuencia de datos medidos en determinados momentos del tiempo, para este caso se dio con relación a los años 1998 hasta el año 2017.

\section{Variables implicadas}

Para la determinación del costo de oportunidad del sector agrícola comercial o transable, se ha utilizado la tasa libre de riesgo, la prima de riesgo de mercado, y el riesgo país (Tabla 6).

CO (Costo de Oportunidad del sector agrícola transable): variable dependiente.

Rf (Tasa libre de riesgo): variable independiente.

Bprima (Riesgo sistemático multiplicado por la prima de riesgo de mercado): variable independiente.

Rpaís (Riesgo País): variable independiente. 
Tabla 7. Análisis de consistencia en la determinación del CO (Costo de oportunidad del sector Agrícola comercial exportador) (BVL, 2017; Banco Central de Reserva del Perú, 2017).

\begin{tabular}{cccccccc}
\hline Año & Rf & Beta & Rm & Prima & Bprima & Rpais & CO \\
\hline 1998 & $11,20 \%$ & 1,00 & $35,40 \%$ & $24,20 \%$ & $24,21 \%$ & $6,55 \%$ & $44,0 \%$ \\
1999 & $11,67 \%$ & 1,00 & $36,40 \%$ & $24,73 \%$ & $24,74 \%$ & $6,04 \%$ & $43,5 \%$ \\
2000 & $11,70 \%$ & 1,42 & $12,88 \%$ & $1,18 \%$ & $1,68 \%$ & $5,68 \%$ & $20,0 \%$ \\
2001 & $11,51 \%$ & 1,27 & $9,00 \%$ & $-2,51 \%$ & $-3,19 \%$ & $6,51 \%$ & $15,5 \%$ \\
2002 & $10,70 \%$ & 1,14 & $10,86 \%$ & $0,16 \%$ & $0,19 \%$ & $6,11 \%$ & $17,4 \%$ \\
2003 & $8,28 \%$ & 0,96 & $21,54 \%$ & $13,26 \%$ & $12,69 \%$ & $4,29 \%$ & $25,6 \%$ \\
2004 & $7,90 \%$ & 0,84 & $25,95 \%$ & $18,04 \%$ & $15,11 \%$ & $3,64 \%$ & $26,9 \%$ \\
2005 & $6,67 \%$ & 0,88 & $26,38 \%$ & $19,71 \%$ & $17,32 \%$ & $2,39 \%$ & $26,6 \%$ \\
2006 & $6,64 \%$ & 0,96 & $42,15 \%$ & $35,51 \%$ & $33,99 \%$ & $1,86 \%$ & $42,7 \%$ \\
2007 & $6,01 \%$ & 1,09 & $41,54 \%$ & $35,53 \%$ & $38,75 \%$ & $1,38 \%$ & $46,4 \%$ \\
2008 & $6,38 \%$ & 1,06 & $32,33 \%$ & $25,95 \%$ & $27,55 \%$ & $2,73 \%$ & $36,9 \%$ \\
2009 & $6,16 \%$ & 1,21 & $38,05 \%$ & $31,89 \%$ & $38,64 \%$ & $2,92 \%$ & $47,9 \%$ \\
2010 & $4,92 \%$ & 1,20 & $40,12 \%$ & $35,20 \%$ & $42,29 \%$ & $1,72 \%$ & $49,1 \%$ \\
2011 & $4,68 \%$ & 1,19 & $36,06 \%$ & $31,38 \%$ & $37,45 \%$ & $1,91 \%$ & $44,2 \%$ \\
2012 & $3,35 \%$ & 1,18 & $34,05 \%$ & $30,70 \%$ & $36,31 \%$ & $1,57 \%$ & $41,4 \%$ \\
2013 & $3,92 \%$ & 1,19 & $30,45 \%$ & $26,53 \%$ & $31,51 \%$ & $1,59 \%$ & $37,2 \%$ \\
2014 & $4,15 \%$ & 1,26 & $28,30 \%$ & $24,15 \%$ & $30,50 \%$ & $1,62 \%$ & $36,4 \%$ \\
2015 & $4,14 \%$ & 1,27 & $24,87 \%$ & $20,73 \%$ & $26,26 \%$ & $2,01 \%$ & $32,5 \%$ \\
2016 & $3,84 \%$ & 1,25 & $26,20 \%$ & $22,37 \%$ & $27,96 \%$ & $2,00 \%$ & $33,9 \%$ \\
2017 & $3,78 \%$ & 1,25 & $23,22 \%$ & $19,44 \%$ & $24,30 \%$ & $1,45 \%$ & $28,7 \%$ \\
\hline
\end{tabular}

Función múltiple:

$\mathrm{CO}_{\text {Agrícola }}=\beta_{1}+\beta_{2} \mathrm{Rf}_{\text {Perú }}+\beta_{3}$ Bprima $\square+\beta_{4}$ RpaisPerú + ui

$\beta 1, \beta 2, \beta 3, \beta 4=$ coeficientes desconocidos de regresión u_i= término de error aleatorio de la regresión

Los resultados obtenidos de la regresión lineal se observan en la Tabla 8 , sobre la cual se reseña brevemente la significancia individual de las variables en las pruebas de hipótesis en adelante.

Una vez logrado el objetivo del estudio en determinar el costo de oportunidad para el sector agrícola comercial exportador del Perú, referente para el período del estudio 1998 - 2017, y de haber revisado la consistencia individual de las variables regresoras y parámetros determinantes se puede confirmar que se cumplen las pruebas de hipótesis alternas, descartando las hipótesis nulas respectiva: La hipótesis alterna, la variable regresora Rf explica el comportamiento de la variable regresada. La variable $\mathrm{Rf}$ ejerce influencia en la variable COAgrícola.

Tabla 8. Regresión lineal de la variable Costo de Oportunidad Agrícola (BVL, 2017).

\begin{tabular}{|c|c|c|c|c|}
\hline \multicolumn{5}{|c|}{$\begin{array}{l}\text { Dependent Variable: COK } \\
\text { Method: Least Squares } \\
\text { Date: 10/01/19 Time: } 22: 53 \\
\text { Sample: } 19982017 \\
\text { Included observations: } 20\end{array}$} \\
\hline Variable & $\begin{array}{r}\text { Coefficie } \\
\text { + }\end{array}$ & Std. Error & t-Statistic & Prob. \\
\hline $\mathrm{C}$ & 0,003833 & 0,011936 & 0,321 & 0,7523 \\
\hline RF & 0,862672 & 0,244503 & 3,528 & 0,0028 \\
\hline BPRIMA & 0,968565 & 0,024268 & 39,910 & 0,0000 \\
\hline RPAIS & 1,311843 & 0,402053 & 3,262 & 0,0049 \\
\hline R-squared & 0,992379 & \multicolumn{2}{|c|}{ Mean dependent var } & 0,341600 \\
\hline Adjusted R-squared & 0,990950 & \multicolumn{2}{|c|}{ S.D. dependent var } & 0,098546 \\
\hline S.E. of regression & 0,009375 & \multirow{2}{*}{\multicolumn{2}{|c|}{$\begin{array}{l}\text { Akaike info criterion } \\
\text { Schwarz criterion }\end{array}$}} & - \\
\hline Sum squared resid & 0,001406 & & & - \\
\hline Log likelihood & 67,24724 & \multicolumn{2}{|c|}{ Hannan-Quinn criter. } & - \\
\hline F-statistic & 694,4861 & \multirow{2}{*}{\multicolumn{2}{|c|}{ Durbin-Watson stat }} & 1,698125 \\
\hline Prob(F-statistic) & 0,000000 & & & \\
\hline
\end{tabular}




\section{$\mathrm{H} 1: \beta_{\mathrm{j}} \neq 0 \quad ; \mathrm{j}=2$,}

La hipótesis alterna, la variable regresora Bprima explica el comportamiento de la variable regresada. La variable Bprima ejerce influencia en la variable $\mathrm{CO}_{\text {Agrícola. }}$

\section{$\mathrm{H}_{1}: \beta_{\mathrm{j}} \neq 0 \quad ; \mathrm{j}=3$,}

La hipótesis alterna, la variable regresora Rpaís explica el comportamiento de la variable regresada. La variable Rpaís ejerce influencia en la variable $\mathrm{CO}_{\text {Agrícola. }}$

\section{$\mathrm{H}_{\mathrm{i}}: \beta_{\mathrm{j}} \neq 0 \quad ; \mathrm{j}=4$,}

El coeficiente del parámetro de la variable Rendimiento de mercado del sector agrícola comercial en el Perú determinó el riesgo sistemático. El análisis estadístico de significancia individual mostró que la variable rendimiento de mercado es significativa y el análisis conjunto arrojó un valor del coeficiente de $\left(\mathrm{R}^{2}\right)$ del $83,15 \%$. Además, el coeficiente beta agrícola peruano, utilizado en el modelo CAPM, determinó el costo de oportunidad del sector agrícola comercial exportador. El análisis estadístico de significancia individual y conjunta arrojó un valor del coeficiente de determinación $\left(\mathrm{R}^{2}\right)$ del $99,24 \%$, teniendo como incidencia un valor de 1,25 para el $\beta$, lo cual mostró mayor riesgo sistemático (volátil) del activo agrario a comparación del mercado, lo cual tiene efecto en la determinación del $\mathrm{CO}_{\text {Agrícola. }}$

De lo anterior, y como resultado de la aplicación del modelo CAPM, se determinó el $\mathrm{CO}_{\text {Agrícola }}$ comercial exportador con el valor de $29,70 \%$, lo que representa un nivel de riesgo de $22,82 \%$ por encima del $6,89 \%$ de la tasa libre de riesgo. Este resultado es consistente con lo esperado para un sector de alto riesgo en el Perú, aunque es importante reconocer que la presencia de sesgo podría llevarnos a conclusiones e interpretaciones un poco asimétricas, en la medida que el retorno o rendimiento de las acciones agrícolas para este estudio está compuesto principalmente por el sub sector azucarero y otros exportables, lo que abre la posibilidades de seguir explorando un indicador de mayor cobertura a otros subsectores agrícolas, esto será posible en la medida que vayan incursionando de manera progresiva en un mercado de capitales en forma abierta y transparente.

De otra parte, uno de los componentes del CAPM trabajado es la beta, obtenido por los métodos estadísticos presentados. Este beta calculado, es un coeficiente básico del riesgo sistemático, que en el caso que se pretenda usarlo para evaluar inversiones a nivel de empresas o unidades productivas, debería considerar ajustar este coeficiente con los niveles de riesgo adicionales o colaterales vinculados a otras variables determinantes de este mismo coeficiente, como es el caso del nivel de endeudamiento que esté afrontando una determinada empresa agrícola y por lo tanto una mayor o menor exposición al riesgo financiero, lo que deberíamos tomar en cuenta la recomendación de un ajuste por la consideración del modelo de Hamada como lo sugieren una serie de autores especializados en el tema de riesgo financiero (Valenzuela, 2012), lo que muy posible y de tener empresas agrícolas con altas tasas de apalancamiento financiero impliquen costo de oportunidad mayor a lo obtenido en nuestros resultados en el presente estudio.

\section{CONCLUSIONES}

Tomando un paquete de acciones empresariales vinculados a la agricultura comercial y exportadora, se logra aproximar un valor del costo de oportunidad para el sector agrícola. El resultado obtenido muestra un Costo de Oportunidad que refleja asumir el riesgo de inversión en este sector, y cuyo resultado se encuentra muy por debajo de las elevadas tasas que aplica la banca comercial a la agricultura, castigando mucho más al sector. Esta diferencia en las tasas del COK agrícola exportador explicaría, por qué muchos proyectos agrícolas evaluados por la banca, con tasas por encima del $40 \%$, comparado con la tasa hallada de $29,70 \%$, restan viabilidad económica a la actividad. En consecuencia, las evaluaciones de las inversiones en el sector agrícola deben realizar un estudio previo en la determinación del Costo de financiamiento con el objetivo de reducir el costo de la deuda a las empresas agrícolas. Así, estas podrían verse incentivadas a realizar o ejecutar mayores proyectos adecuadamente evaluados. 


\section{Conflictos de intereses}

Los autores firmantes del presente trabajo de investigación declaran no tener ningún potencial conflicto de interés personal o económico con otras personas $\mathrm{u}$ organizaciones que puedan influir indebidamente con el presente manuscrito.

\section{Contribuciones de los autores}

Preparación y ejecución: FM, CC; Desarrollo de la metodología: FM, CC; Concepción y diseño: FM, CC; Edición del artículo: FM, CC; Supervisión del estudio: FM, CC.

\section{LITERATURA CITADA}

- Banco Central de Reserva del Perú. (2017). Serie estadísticas / Mercado de Capitales / Índices bursátiles, varios años. Disponible en http://www.bcrp.gob.pe/2017

- BVL [Bolsa de Valores de Lima]. (2017). Histórico de cotizaciones de acciones del sector agrario, base de datos estadística. Disponible en http://www.bvl.com.pe/2017

- Fernández, J. (2008). Internalización del riesgo en la determinación de la tasa de costo de capital. España: Universidad de Rioja, España.
- Kristjanpoller, W., \& Liberona, C. (2010). Comparación de modelos de predicción de retornos accionarios en el Mercado Accionario Chileno: CAPM, FAMA y FRENCH y REWARD BETA. EconoQuantum, 7(1): 121140.

- Markowitz, H. (1952). Portfolio Selection.The Journal of Finance, 7(1), 77-91. Disponible en https://www.math.ust.hk/ maykwok/courses/m a362/07F/markowitz_JF.pdf

- MINAGRI [Ministerio de Agricultura y Riego]. (2017). Expansión de la Frontera Agrícola en el Perú. Disponible en http://www.minagri.gob.pe/2017

- Sarmiento, A. (2008). Análisis de costos de la generación de energía eléctrica mediante fuentes renovable en el sistema eléctrico colombiano. Colombia: Universidad Militar Nueva Granada.

- Valenzuela, A. (2012). Costo de Capital, modelo CAPM. Chile: Facultad de Ciencias Sociales y Económicas, Universidad Católica de Maule, Maule.

- Vélez-Pareja, I. (2011). Una nota sobre el costo promedio de capital. Universidad Complutense de Madrid, España: 